\title{
KAGRA: 2.5 generation interferometric gravitational wave detector
}

\author{
KAGRA collaboration
}

\begin{abstract}
The recent detections of gravitational waves (GWs) reported by the LIGO and Virgo collaborations have made a significant impact on physics and astronomy. A global network of GW detectors will play a key role in uncovering the unknown nature of the sources in coordinated observations with astronomical telescopes and detectors. Here we introduce KAGRA, a new GW detector with two $3 \mathrm{~km}$ baseline arms arranged in an 'L' shape. KAGRA's design is similar to the second generations of Advanced LIGO and Advanced Virgo, but it will be operating at cryogenic temperatures with sapphire mirrors. This low-temperature feature is advantageous for improving the sensitivity around $100 \mathrm{~Hz}$ and is considered to be an important feature for the third-generation GW detector concept (for example, the Einstein Telescope of Europe or the Cosmic Explorer of the United States). Hence, KAGRA is often called a 2.5-generation GW detector based on laser interferometry. KAGRA's first observation run is scheduled in late 2019, aiming to join the third observation run of the advanced LIGO-Virgo network. When operating along with the existing GW detectors, KAGRA will be helpful in locating GW sources more accurately and determining the source parameters with higher precision, providing information for follow-up observations of GW trigger candidates.
\end{abstract}

S eeing is believing. We were reminded of this proverb when we received the news of the discovery of GW150914, the first direct detection of gravitational waves $(\mathrm{GWs})^{1}$. The existence of GWs has been believed since Russel Hulse and Joseph Taylor discovered the binary pulsar PSR B1913 + 16 in 1974 (ref. ${ }^{2}$ ). The longterm radio observation of this system has shown that the observed orbital decay is well described by the energy/angular momentum loss due to GW emission as predicted by Einstein in 1915 (ref. ${ }^{3}$ ). However, the direct detection of GWs had an extraordinary impact not only on the scientific community but also on the general public.

The first five GW sources ${ }^{1,4-7}$ were identified to be binary black holes (BHs). Their masses range between 20 and $60 M_{\odot}$, which is heavier than known stellar BHs in our Galaxy. In addition to the confirmation of the existence of binary BHs, which is one of the scientific achievements of GW detection, more GW observations will allow us to better understand the formation and evolution of binary BHs.

The latest event GW170817 (ref. ${ }^{8}$ ) was a long-sought-after binary neutron star (NS) merger event. The distance and location of GW170817 were narrowed down to $40 \pm 8 \mathrm{Mpc}$ and about $30 \mathrm{deg}^{2}$ in the sky by the LIGO-Virgo ${ }^{9,10}$ observation, allowing astronomers to identify electromagnetic counterparts and the host galaxy (NGC $4993)^{11}$. Furthermore, afterglows from the merger remnants and later outcomes via various baryonic interactions were observed by the telescopes on Earth as well as space satellites from radio to $\gamma$-rays.

All these discoveries are success stories of long-baseline laser interferometers as a highly effective tool to explore the Universe via GWs. LIGO consists of two $4 \mathrm{~km}$ laser interferometers in Livingston, Louisiana and Hanford, Washington in the United States. Virgo is a $3 \mathrm{~km}$ interferometer located in Pisa, Italy. Coincident signal-extraction analyses of these three detectors can eliminate false detections due to noise, and by using triangulation, the source location in the sky can be determined within several tens of square degrees. For a more precise source localization and source parameter estimation, it is essential to extend the global network of GW detectors, with KAGRA being the next to come online.
Figure 1 shows the location of KAGRA in Kamioka, Japan. The interferometer shares the area with the well-known neutrino detectors Super-Kamiokande and KamLAND. Kamioka is a small town located 1.5 hour driving distance from the city of Toyama, with its biggest claim to fame being an old mine.

Compared with existing laser interferometers, KAGRA is technologically unique in two features. Firstly, it is located in an underground site to reduce seismic noise. Secondly, KAGRA's test masses are sapphire mirrors that are designed to be operated at cryogenic temperatures $(20 \mathrm{~K})$ to reduce thermal noise. KAGRA is a resonant sideband extraction interferometer ${ }^{12}$, and quantum non-demolition techniques ${ }^{13}$ are planned to be applied to beat the standard quantum limit of displacement measurements. As a result, KAGRA is expected to reach a sensitivity equivalent to those of Advanced LIGO and Advanced Virgo; that is, $2 \times 10^{-24} \sqrt{\mathrm{Hz}}-1$ at $100 \mathrm{~Hz}$.

\section{Milestones of KAGRA construction and operations}

In Japan, plans to construct interferometric GW detectors started in the 1980s. In the early 1990s, the Institute of Space and Astronautical Science (ISAS) and the National Astronomical Observatory of Japan (NAOJ) constructed a $100 \mathrm{~m}$ delay-line Michelson interferometer (TENKO-100) ${ }^{14}$ and a $2 \mathrm{~m}$ Fabry-Perot Michelson interferometer ${ }^{15}$, respectively. TENKO-100 realized light paths that were 102 times longer than the arm length (the equivalent of a $10.2 \mathrm{~km}$ arm length) and reached a sensitivity of $1.1 \times 10^{-19} \sqrt{\mathrm{Hz}}-1$ in the frequency range of $800 \mathrm{~Hz}-2.5 \mathrm{kHz}$.

In 1995, the construction of a 300 m Fabry-Perot Michelson interferometer, called TAMA (or TAMA300) ${ }^{16}$, began in the Mitaka campus of the NAOJ. The name TAMA originated from the area where $\mathrm{NAOJ}$ is located. After three years of commissioning, the TAMA interferometer was operated for the first time in 1998 with a sensitivity of $5 \times 10^{-21} \sqrt{\mathrm{Hz}}^{-1}$. In 2000, the $40 \mathrm{~m}$ prototype of LIGO was built, but the 1998 sensitivity achieved by TAMA remained unbeaten.

In 2001, TAMA was successfully operated for more than 1,000 hours (ref. ${ }^{17}$ ) and in 2002, it also took part in a joint operation with LIGO's second science run (S2) for two months. TAMA was 
a

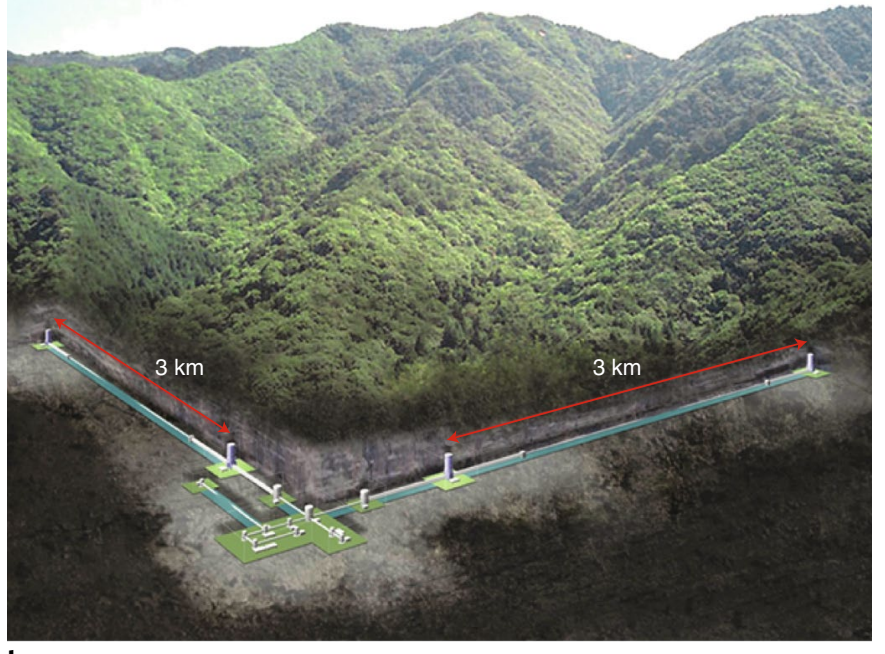

b

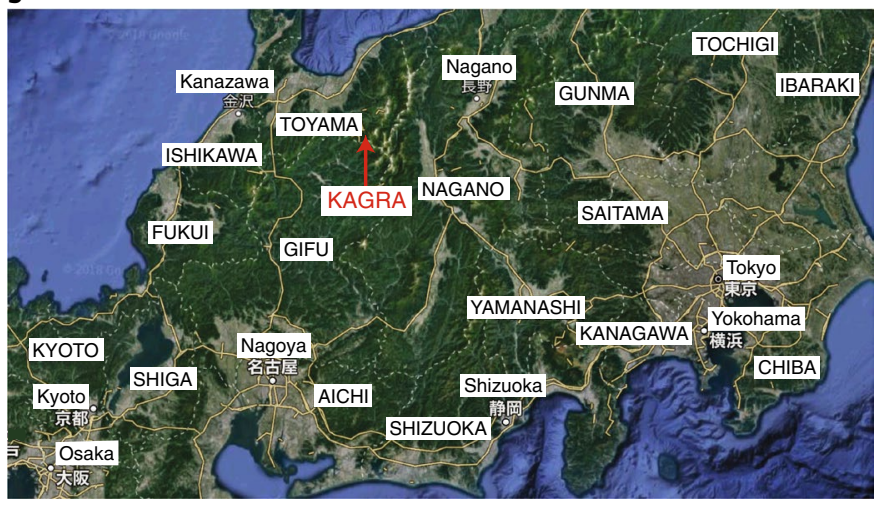

Fig. 1 | Location of KAGRA. a, Concept image of KAGRA: a 3 km cryogenic interferometer inside Ikenoyama mountain. b, KAGRA is located in Kamioka, Gifu, Japan, which is located 220 km northwest of Tokyo. Credit: 2018 Data SIO, NOAA, US Navy, NGA, GEBCO, Data Japan Hydrographic Association, Landsat/Copernicus, Map data (C2018 Google, ZENRIN

planned as a prototype to develop future technologies for a kilometre-scale interferometer that included a power-recycling system and a seismic attenuation system. TAMA's final (and best) sensitivity of $1.3 \times 10^{-21} \sqrt{\mathrm{Hz}}-1$ was obtained at around $1 \mathrm{kHz}$.

TAMA was located in the city of Mitaka, a suburb of Tokyo. In the frequency band below $100 \mathrm{~Hz}$, therefore, significant seismic noise due to human activities in and around the mega city was inevitable. To overcome the large seismic noise, it was decided to put a planned future interferometer underground. An old mine in a mountain in Kamioka was selected as the site of this new interferometer and experiments for early commissioning began. The Laser Interferometer Small Observatory in a Mine (LISM) ${ }^{18}$ project (2000-2002) brought a $20 \mathrm{~m}$ Fabry-Perot interferometer from NAOJ to Kamioka and confirmed that the Kamioka site is less affected by seismic noise than the Tokyo/Mitaka area. The LISM and TAMA groups performed a simultaneous observation and the first veto analysis, which aims to remove false triggers caused by the instrument ${ }^{19,20}$.

The Cryogenic Laser Interferometer Observatory (CLIO) ${ }^{21}$ was constructed next in Kamioka from 2002. CLIO was an interferometer with two perpendicular $100 \mathrm{~m}$ arms and sapphire mirrors were installed and cooled down to $20 \mathrm{~K}$. The operation started in 2005 and the experiments continued until 2010. This system reduced various thermal noises and the seismic noise was two orders of magnitude lower than that of the Tokyo area.
Although various experiments showcased the possible scientific achievements of the project and the plausibility of fundamental technologies, the proposal for developing a kilometre-scale cryogenic GW detector remained in limbo for many years. This was mainly due to the fact that there was no GW detection reported in 2000s. Without a detection, the proposed kilometre-baseline interferometer concept Large-scale Cryogenic Gravitational wave Telescope (LCGT) 22 was considered to be too expensive and too risky. The wind started to blow favourably when Takaaki Kajita became the director of the Institute for Cosmic Ray Research (ICRR, University of Tokyo) in 2008. Realizing its importance, he decided to lead the GW project by his own account, which was the starting point of the current project. The LCGT project was finally approved in 2010 with a starting budget of JP¥14 billion (US\$150 million) for construction, and the excavation of the tunnels in Kamioka began in 2012, after an one-year delay due to the 2011 Tohoku earthquake. During the construction, LCGT was given its nickname, KAGRA, chosen from a public naming contest. The name KAGRA is taken from KAmioka (the location) plus GRAvity; it is also similar to the Japanese word kagura, which is a type of traditional sacred dance accompanied by music dedicated to gods.

After a two-year excavation and another two-year facility installation period, KAGRA performed a test operation in March and April 2016 with a simple $3 \mathrm{~km}$ Michelson interferometer configuration, called iKAGRA (initial KAGRA) ${ }^{23}$.

The strain sensitivity of iKAGRA was limited by seismic noise below $3 \mathrm{~Hz}$, by acoustic noise over $100 \mathrm{~Hz}$ to $3 \mathrm{kHz}$, and by sensor noise at 3-5 kHz. Unfortunately, a series of large earthquakes hit the Kumamoto area during the period of iKAGRA operations. Such noise sources were not avoidable with the iKAGRA configuration, but iKAGRA still provided the collaboration with invaluable experience in controlling the kilometre-scale laser interferometer with unprecedented sensitivity.

\section{Current status of KAGRA}

Immediately after the iKAGRA operation, the KAGRA collaboration put great effort into improving the whole system and spent two more years installing cryogenic facilities and upgrading vibrationisolation systems and high vacuum systems. This period is called phase 1 of bKAGRA (baseline KAGRA). KAGRA is the world's third-largest vacuum system; the first two are LIGO-Livingston and LIGO-Handford.

One of the major upgrades is the vacuum system; KAGRA now has the world's tallest vibration-isolation systems $(13.5 \mathrm{~m})$, which helps to reduce seismic noise at low frequencies. Two $23 \mathrm{~kg}$ sapphire mirrors have been installed at both ends, and one of them was kept at $18 \mathrm{~K}$ for 30 days continuously.

Due to a leakage of the vacuum that was found in April 2018, the experimental operation was delayed for five days, but the phase 1 operation was successfully undertaken for 9 days from 28 April to 6 May 2018. The duty cycle of the first five days of the phase 1 operation reached $88.6 \%$ between 28 April and 2 May, while the duty cycle on 3 and 4 May dropped to $26.8 \%$, and slightly improved to $59.8 \%$ on the final days (5 and 6 May). The low duty cycle on 3 May and afterwards were mainly attributed to the micro-seismic noise caused by a heavy storm, local earthquakes and volcanic eruptions in Hawaii. The obtained sensitivity during phase 1 was worse than the final sensitivities of TAMA and CLIO, apart from the lower frequencies, where KAGRA's sensitivity was indeed better than TAMA (see the designed sensitivity of bKAGRA in Fig. 2). More detailed results of the phase 1 operation will be reported elsewhere.

On 7 May 2018, the KAGRA collaboration announced the beginning of phase 2 and has been working on the installations/upgrades of more instruments, such as additional optics and a new higherpower laser source. 


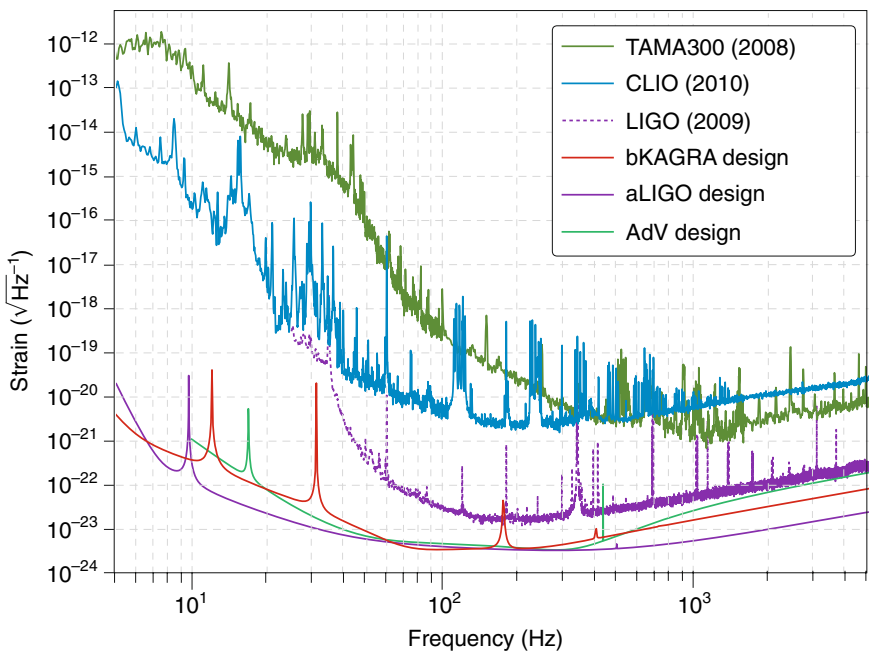

Fig. 2 | Evolution of GW detectors in Japan. We compare the measured sensitivity curves obtained from TAMA300 (2008) and CLIO (2010) with the design sensitivity of bKAGRA, which is to be accomplished in 2022 in the current plan. The sensitivity curves of LIGO during the science run in 2009-2010 and of Advanced LIGO (aLIGO)/Advanced Virgo (AdV) designs are also shown.

Since the beginning of the iKAGRA commissioning, one of the important goals of the KAGRA collaboration has been to contribute to the international efforts for GW detection. As shown by the LIGO-Virgo joint observations of GW170814 and GW170817, independent detections at different sites on Earth will be not only useful for increasing the signal-to-noise ratio of a GW trigger signal but also important to better constrain the GW source location. Increasing the number of detectors is also intrinsically essential to distinguish polarization modes of GWs. When KAGRA is added to the global network of GW detectors, the total network sensitivity will be sufficient to search for signatures due to non-tensorial GWs and to more stringently test general relativity ${ }^{24}$.

With this in mind, there have been calls from both within and outside of the collaboration to take part in joint observations. To take advantage of a quadrupole detector network around the Earth in the search for GWs, the KAGRA collaboration has been investing great effort to accelerate the commissioning schedule shown in the 'scenario paper $^{25}$. If everything goes well, KAGRA will join LIGOVirgo's third observation run (O3), which is planned to start from February 2019 and last for a year.

The latest road map of KAGRA's GW observation is presented in Fig. 3, along with the timeline of the LIGO-Virgo observation plans. This figure is an update of Fig. 2 in ref. ${ }^{25}$. By March 2019, almost all the optics for the final interferometer configuration are to be installed. After some tuning of the interferometer, KAGRA plans to begin its observation phase no later than October 2019. If resonant sideband extraction implementation is ready, then KAGRA will start with a $25 \mathrm{Mpc}$ observational range in NS-NS binary coalescence, a distance that is at the same level of Virgo at the end of O2. While waiting for the interferometer to be operational, the data analysis groups in KAGRA are planning to start co-data analysis with the LIGO and Virgo groups from the beginning of O3. After O3, KAGRA will also try to catch up with LIGO-Virgo's $\mathrm{O} 4$ schedule from its beginning. The main interferometer components are planned to be installed by March 2019 , and for O4, we expect to approach the designed sensitivity at a horizon distance of $130 \mathrm{Mpc}$ for NS-NS binary inspirals in the broadband configuration.

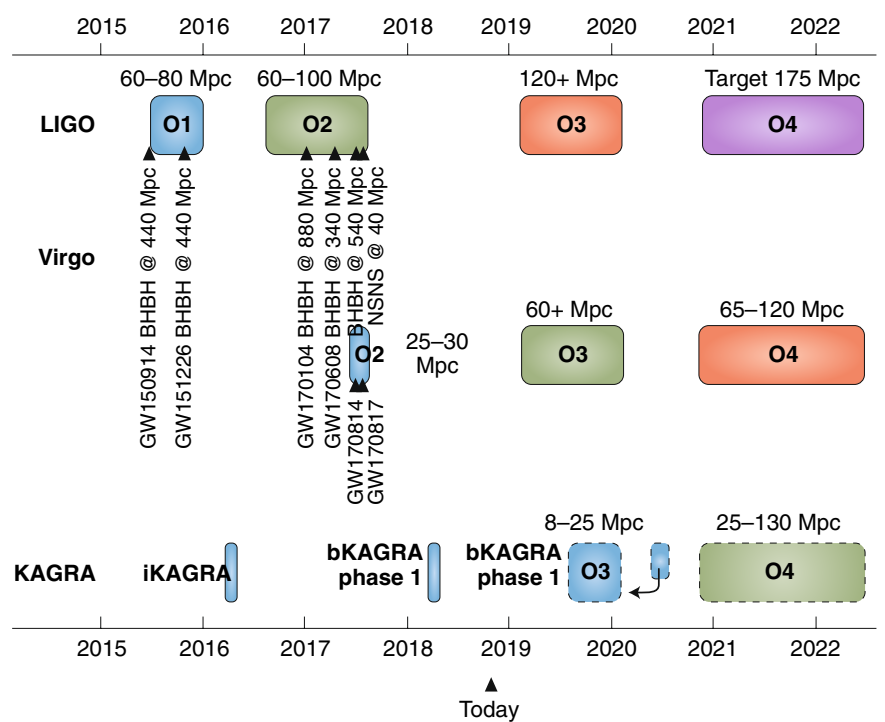

Fig. 3 | Observation plans of LIGO, VIRGO and KAGRA. An unofficial update of Fig. 2 presented in ref. ${ }^{25}$. The coloured bars indicate the first (blue), second (green) and third (red) observation periods. The details of KAGRA's long-term plan are still under discussion. In principle, KAGRA's first observation run will coincide with the later phase of $\mathrm{O} 3$ by the LIGO-Virgo network. The KAGRA collaboration aims to begin its observation run from the beginning of $\mathrm{O} 4$, in coordination with $\mathrm{LIGO}$ and VIRGO.

\section{KAGRA international collaboration}

The KAGRA collaboration is by all means international. As of October 2018, the collaboration consists of more than 200 researchers from 90 institutions in 15 countries and regions. The KAGRA collaboration has a scientific decision-making body, named KAGRA Scientific Congress (KSC). KSC organizes the KAGRA international workshops outside of Japan twice every year and interacts with the broader scientific community.

KSC is also setting the organization for future joint observations, and is now re-organizing data analysis groups to match their structures with those of LIGO and Virgo groups. Data analysis teams are preparing the original analysis codes, called 'KAGRA Algorithmic Library' (KAGALI); kagari is a Japanese word for the bonfire at a celebration party. The code includes, for example, a couple of new methods for extracting ring-down waveform solely with the purpose of testing gravity theories, and such new ideas are expected to enhance the advantages of joint observations with LIGO and Virgo.

Including the ELiTES (ET-LCGT Telescopes: Exchange of Scientists) project in 2012-2017, the KAGRA collaboration has been trying to expand international collaboration. For example, the 'core-to-core' (C2C) programme funded by the Japan Society for the Promotion of Science (JSPS) is dedicated to human resource exchanges and organizing academic meetings relevant to KAGRA and GW science.

In addition to local computing centers at Kamioka Observatory, ICRR, Osaka City University and so on, the KAGRA collaboration runs two mirror sites for data storage in Korea (Korea Institute of Science and Technology Information) and in Taiwan (Academia Sinica). KISTI and Academia Sinica also provide computing resources for data analysis.

\section{KAGRA's future}

The LIGO and Virgo collaborations have plans to upgrade their detectors to $\mathrm{A}+\left(\right.$ ref. ${ }^{26}$ ) and $\mathrm{AdV}+\left(\right.$ ref. ${ }^{27}$ ) to improve the sensitivity by a factor of roughly two compared with the original Advanced LIGO and Advanced Virgo designs. For example, both $\mathrm{A}+$ and 
$\mathrm{AdV}+$ detectors will incorporate frequency-dependent squeezing to reduce quantum noise and lower-loss coating to reduce coating thermal noise.

Similarly, the KAGRA collaboration have recently started planning for an upgrade of KAGRA to realize a binary neutron-star range of more than $150 \mathrm{Mpc}$. KAGRA has the potential to improve the sensitivity because of its cryogenic operation and lower seismic noise compared with LIGO and Virgo. Completion of the A+ and AdV+ upgrades is expected by $\sim 2023$, and the KAGRA collaboration aims for a similar timeline for KAGRA.

Cryogenic operation and underground construction are expected to be key technologies of the third-generation large-scale GW detectors ${ }^{28,29}$. For example, collaborative research and development activities between KAGRA and the next-generation projects, as represented by the ELiTES project, have been supported by the Japanese government and the European Commission in 2012-2017.

The realization of the KAGRA project is made possible by enormous support from the scientific community as well as by great effort from researchers around the world. The upgraded bKAGRA is about to see the 'first light' in 2019. Nobody doubts the importance of GW research in astronomy, physics and also in engineering. We believe KAGRA will definitely contribute to these fields, especially to GW science, and help to broaden our understanding of gravity and of the Universe.

Received: 14 November 2018; Accepted: 19 November 2018; Published online: 8 January 2019

\section{References}

1. Abbott, B. P. et al. Observation of gravitational waves from a binary black hole merger. Phys. Rev. Lett. 116, 061102 (2016).

2. Hulse, R. A. \& Taylor, J. H. Discovery of a pulsar in a binary system. Astrophys. J. 195, L51 (1975).

3. Taylor, J. H. \& Weisberg, J. M. Gravitational radiation and the binary pulsar Astrophys. J. 253, 908 (1981).

4. Abbott, B. P. et al. GW151226: observation of gravitational waves from a 22-solar-mass binary black hole coalescence. Phys. Rev. Lett. 116 241103 (2016).

5. Abbott, B. P. et al. GW170104: observation of a 50-solar-mass binary black hole coalescence at redshift 0.2. Phys. Rev. Lett. 118, 221101 (2017).

6. Abbott, B. P. et al. GW170608: observation of a 19 solar-mass binary black hole coalescence. Astrophys. J. Lett. 851, L35 (2017).

7. Abbott, B. P. et al. GW170814: a three-detector observation of gravitational waves from a binary black hole coalescence. Phys. Rev. Lett. 119, 141101 (2017).

8. Abbott, B. P. et al. GW 170817: observation of gravitational waves from a binary neutron star inspiral. Phys. Rev. Lett. 119, 161101 (2017).

9. Aasi, J. et al. Advanced LIGO. Class. Quantum Gravity 32, 074001 (2015)

10. Acernese, F. et al. Advanced Virgo: a second-generation interferometric gravitational wave detector. Class. Quantum Gravity 32, 024001 (2015).

11. Abbott, B. P. et al. Multi-messenger observations of a binary neutron star merger. Astrophys. J. Lett. 848, L12 (2017).

12. Mizuno, J. et al. Resonant sideband extraction: a new configuration for interferometric gravitational wave detectors. Phys. Lett. A 175, 273 (1993).

13. Somiya, K. Detector configuration of KAGRA - the Japanese cryogenic gravitational-wave detector. Class. Quantum Gravity 29, 124007 (2012).

14. Kawashima, K. Laser Interferometer (TENKO-10 and -100) for Gravitational Wave Antenna Development Report No. 640 (Institute of Space and Astronautical Science, 1991).
15. Sato, S. et al. High-gain power recycling of a Fabry-Perot Michelson interferometer for a gravitational-wave antenna. Appl. Opt. 36, 1446 (1997).

16. Tsubono, K. in Gravitational Wave Experiments (eds Coccia, E., Pizzella, G. \& Ronga F.) 112-114 (World Scientific, Singapore, 1995).

17. Ando, M. et al. Stable operation of a 300-m laser interferometer with sufficient sensitivity to detect gravitational-wave events within our Galaxy. Phys. Rev. Lett. 86, 3950 (2001).

18. Sato, S. et al. Ultrastable performance of an underground-based laser interferometer observatory for gravitational waves. Phys. Rev. D 69, 102005 (2014).

19. Ando, M. et al. Analysis methods for burst gravitational waves with TAMA data. Class. Quantum Gravity 21, S1679 (2004).

20. Christensen, N. et al. LIGO S6 detector characterization studies. Class. Quantum Gravity 27, 194010 (2010).

21. Uchiyama, T. et al. Reduction of thermal fluctuations in a cryogenic laser interferometric gravitational wave detector. Phys. Rev. Lett. 108, 141101 (2012).

22. Kuroda, K. et al. Large-scale cryogenic gravitational wave telescope. Int. J. Mod. Phys. D 8, 557 (1999).

23. Akutsu, T. et al. Construction of KAGRA: an underground gravitational-wave observatory. Prog. Theor. Exp. Phys. 2018, 013 F01 (2018).

24. Takeda, H. et al. Polarization test of gravitational waves from compact binary coalescences. Phys. Rev. D 98, 022008 (2018).

25. Abbot, B. P. et al. Prospects for observing and localizing gravitational-wave transients with Advanced LIGO, Advanced Virgo and KAGRA. Living Rev. Relativ. 21, 3 (2018).

26. Miller, J. et al. Prospects for doubling the range of Advanced LIGO. Phys. Rev. D 91, 062005 (2015).

27. Degallaix, J. Advanced Virgo+ Preliminary Studies VIR-0300A-18 (Virgo Collaboration, 2018)

28. Punturo, M. et al. The Einstein Telescope: a third-generation gravitational wave observatory. Class. Quantum Gravity 27, 194002 (2010).

29. Abbott, B. P. et al. Exploring the sensitivity of next generation gravitational wave detectors. Class. Quantum Gravity 34, 044001 (2017).

\section{Acknowledgements}

This work was supported by MEXT, JSPS Leading-edge Research Infrastructure Program, JSPS Grant-in-Aid for Specially Promoted Research 26000005, JSPS Grantin-Aid for Scientific Research on Innovative Areas 2905: JP17H06358, JP17H06361 and JP17H06364, JSPS Core-to-Core Program (A. Advanced Research Networks), JSPS Grant-in-Aid for Scientific Research (S) 17H06133, the joint research programme of the Institute for Cosmic Ray Research, University of Tokyo, National Research Foundation (NRF) grant of Korea and Computing Infrastructure Project of KISTI-GSDC in Korea, the LIGO project, and the Virgo project. The authors appreciate M. Karouzos for his kind feedback to improve the draft.

\section{Author contributions}

C. Kim, Y. Michimura, H. Shinkai and A. Shoda were responsible for writing the first draft of the manuscript and incorporated comments from the collaboration in the course of completing the manuscript. All members of the KAGRA collaboration were responsible for the scope and scientific facts of the manuscript, including data analysis and the resulting figures.

\section{Competing interests}

The authors declare no competing interests.

\section{Additional information}

Reprints and permissions information is available at www.nature.com/reprints.

Correspondence should be addressed to C.K., Y.M., H.S. or A.S.

Publisher's note: Springer Nature remains neutral with regard to jurisdictional claims in published maps and institutional affiliations.

(c) Springer Nature Limited 2019 
KAGRA collaboration

T. Akutsu', ${ }^{1,2}$, M. Ando ${ }^{1,3,4}$, K. Arai ${ }^{5}$, Y. Arai ${ }^{5}$, S.Araki ${ }^{6}$, A. Araya7, N. Aritomi ${ }^{3}$, H. Asada ${ }^{8}$, Y.Aso ${ }^{9,10,}$ S. Atsuta" ${ }^{11}$ K. Awai ${ }^{12}$, S. Bae ${ }^{13}$, L. Baiotti' ${ }^{14}$, M. A. Barton', K. Cannon ${ }^{4}$, E. Capocasa', C-S. Chen ${ }^{15}$, T-W.Chiu ${ }^{15}$, K. Choo', Y-K.Chu' ${ }^{15}$, K. Craig ${ }^{5}$, W. Creus ${ }^{17}$, K. Doi ${ }^{18}$, K. Eda ${ }^{4}$, Y.Enomoto ${ }^{3}$, R. Flaminio ${ }^{1,19}$, Y. Fujii' ${ }^{20}$, M.-K. Fujimoto', M. Fukunaga ${ }^{5}$, M. Fukushimaa ${ }^{2}$, T. Furuhata ${ }^{18}$, S. Haino ${ }^{17}$, K. Hasegawa ${ }^{5}$, K. Hashino ${ }^{18}$, K. Hayama ${ }^{21}$, S. Hirobayashi2 ${ }^{22}$, E. Hirose ${ }^{5}$, B. H. Hsieh ${ }^{23}$, C-Z. Huang ${ }^{15}$, B. Ikenoue ${ }^{2}$, Y. Inoue ${ }^{17}$, K. Ioka ${ }^{24}$, Y.Itoh ${ }^{25}$, K. Izumi ${ }^{26}$, T. Kaji ${ }^{25}$, T. Kajita ${ }^{27}$, M. Kakizaki ${ }^{18}$, M. Kamiizumi' ${ }^{12}$, S. Kanbara ${ }^{18}$, N. Kanda ${ }^{25}$, S. Kanemura ${ }^{14}$, M. Kaneyama ${ }^{25}$, G. Kang ${ }^{13}$, J. Kasuya", Y.Kataoka"1, N. Kawai" ${ }^{11}$ S. Kawamura' ${ }^{12}$, T. Kawasaki ${ }^{3}$, C. Kim ${ }^{28 \star}$, J.Kim ${ }^{29}$, J.C. Kim ${ }^{30}$, W. S. Kim ${ }^{31}$, Y.-M. Kim ${ }^{32}$, N. Kimuraa ${ }^{33}$, T.Kinugawa ${ }^{5}$, S. Kirii' ${ }^{12}$, Y. Kitaoka ${ }^{25}$, H. Kitazawa ${ }^{18}$, Y. Kojima ${ }^{34}$, K. Kokeyama ${ }^{12}$, K. Komori ${ }^{3}$, A. K.H.Kong ${ }^{35}$, K. Kotake ${ }^{21}$, R. Kozu ${ }^{36}$, R. Kumar ${ }^{37}$, H-S. Kuo ${ }^{15}$, S. Kuroyanagi ${ }^{38}$, H.K. Lee ${ }^{39}$, H.M. Lee ${ }^{40}$, H. W. Lee ${ }^{30}$, M. Leonardi', C-Y.Lin ${ }^{41}$, F-L. Lin ${ }^{4,15}$, G.C. Liu ${ }^{42}$, Y. Liu ${ }^{43}$, E. Majorana ${ }^{44}$, S. Mano ${ }^{45}$, M. Marchio', T. Matsui ${ }^{46}$, F. Matsushima ${ }^{18}$, Y. Michimura ${ }^{3 \star}$, N. Mio ${ }^{47}$, O. Miyakawa ${ }^{12}$, A. Miyamoto ${ }^{25}$, T. Miyamoto ${ }^{36}$, K. Miyo ${ }^{12}$, S.Miyoki' ${ }^{12}$, W. Morii ${ }^{48}$, S. Morisaki ${ }^{4}$, Y.Moriwaki ${ }^{18}$, T.Morozumi ${ }^{5}$, M.Musha ${ }^{49}$, K.Nagano ${ }^{5}$, S. Nagano ${ }^{50}$, K.Nakamura', T. Nakamura ${ }^{51}$, H.Nakano ${ }^{52}$, M.Nakano ${ }^{5}$, K.Nakao ${ }^{25}$, T.Narikawa ${ }^{51}$, L. Naticchioni ${ }^{44}$, L. Nguyen Quynh ${ }^{53}$, W.-T. Ni ${ }^{35,54,55}$, A. Nishizawa ${ }^{38}$, Y. Obuchi ${ }^{2}$, T. Ochi ${ }^{5}$, J. J. Oh ${ }^{31}$,

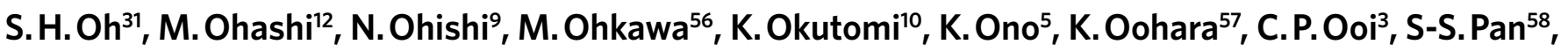
J. Park ${ }^{16}$, F. E. Peña Arellanoo' ${ }^{12}$ I.Pinto ${ }^{59}$, N. Sago ${ }^{60}$, M. Saijo ${ }^{61}$, S. Saitou ${ }^{2}$, Y.Saito ${ }^{12}$, K. Sakai ${ }^{62}$, Y. Sakai ${ }^{57}$, Y.Sakai ${ }^{3}$, M.Sasai ${ }^{25}$, M. Sasaki ${ }^{63}$, Y. Sasaki ${ }^{64}$, S.Sato ${ }^{65}$, N. Sato ${ }^{2}$, T. Sato ${ }^{56}$, Y. Sekiguchi ${ }^{66}$, N. Seto ${ }^{51}$, M. Shibata ${ }^{24}$, T. Shimoda ${ }^{3}$, H. Shinkai ${ }^{67 \star}$, T. Shishido ${ }^{68}$, A.Shoda ${ }^{1 \star}$, K. Somiya ${ }^{11}$, E. J. Son ${ }^{31}$, A. Suemasa ${ }^{49}$, T.Suzuki ${ }^{56}$, T. Suzuki ${ }^{5}$, H. Tagoshi ${ }^{5}$, H. Tahara ${ }^{20}$, H. Takahashi ${ }^{64}$, R. Takahashi', A. Takamori', H. Takeda ${ }^{3}$, H. Tanaka ${ }^{23}$, K. Tanaka ${ }^{25}$, T. Tanaka ${ }^{51}$, S. Tanioka ${ }^{1,10}$, E. N. Tapia San Martin', D. Tatsumi', T. Tomaru ${ }^{33}$, T. Tomura ${ }^{12}$, F. Travasso ${ }^{69}$, K. Tsubono ${ }^{3}$, S. Tsuchida ${ }^{25}$, N. Uchikata ${ }^{70}$, T.Uchiyama ${ }^{12}$, T.Uehara ${ }^{71,72}$, S.Ueki ${ }^{64}$, K.Ueno ${ }^{4}$, F. Uraguchi ${ }^{2}$, T. Ushiba ${ }^{5}$, M. H.P. M.van Putten ${ }^{73}$, H. Vocca ${ }^{69}$, S. Wada ${ }^{3}$, T. Wakamatsu ${ }^{57}$, Y. Watanabe ${ }^{57}$, W-R.X u $^{15}$, T. Yamada ${ }^{23}$, A. Yamamoto ${ }^{6}$, K. Yamamoto ${ }^{18}$, K. Yamamoto ${ }^{23}$, S. Yamamoto67, T. Yamamoto ${ }^{12}$, K. Yokogawa ${ }^{18}$, J. Yokoyama ${ }^{3,4,20}$, T. Yokozawa ${ }^{12}$, T. H. Yoon ${ }^{74}$, T. Yoshioka ${ }^{18}$, H. Yuzurihara' ${ }^{5}$ S. Zeidler ${ }^{1}$ and Z.-H. Zhu ${ }^{75}$

'National Astronomical Observatory of Japan (NAOJ), Mitaka, Tokyo, Japan. ${ }^{2}$ Advanced Technology Center, National Astronomical Observatory of Japan (NAOJ), Mitaka, Tokyo, Japan. ${ }^{3}$ Department of Physics, The University of Tokyo, Bunkyo-ku, Tokyo, Japan. ${ }^{4}$ Research Center for the Early Universe (RESCEU), The University of Tokyo, Bunkyo-ku, Tokyo, Japan. ${ }^{5}$ Institute for Cosmic Ray Research (ICRR), KAGRA Observatory, The University of Tokyo, Kashiwa, Chiba, Japan. ${ }^{6}$ Accelerator Laboratory, High Energy Accelerator Research Organization (KEK), Tsukuba, Ibaraki, Japan. ${ }^{7}$ Earthquake Research Institute, The University of Tokyo, Bunkyo-ku, Tokyo, Japan. ${ }^{8}$ Department of Mathematics and Physics, Hirosaki University, Hirosaki, Aomori, Japan. ${ }^{9}$ Kamioka Branch, National Astronomical Observatory of Japan (NAOJ), Hida, Gifu, Japan. ${ }^{10}$ The Graduate University for Advanced Studies (SOKENDAI), Mitaka, Tokyo, Japan. "'Graduate School of Science and Technology, Tokyo Institute of Technology, Meguro-ku, Tokyo, Japan. ${ }^{12}$ Institute for Cosmic Ray Research (ICRR), KAGRA Observatory, The University of Tokyo, Hida, Gifu, Japan. ${ }^{13}$ Korea Institute of Science and Technology Information (KISTI), Daejeon, Korea. ${ }^{14}$ Graduate School of Science, Osaka University, Toyonaka, Osaka, Japan. ${ }^{15}$ Department of Physics, National Taiwan Normal University, Taipei, Taiwan. ${ }^{16}$ Department of Physics, Sogang University, Seoul, Korea. ${ }^{17}$ Institute of Physics, Academia Sinica, Taipei, Taiwan. ${ }^{18}$ Department of Physics, University of Toyama, Toyama, Toyama, Japan. ${ }^{19}$ Laboratoire d'Annecy de Physique des Particules (LAPP), Univ. Grenoble Alpes, Université Savoie Mont Blanc, CNRS/IN2P3, F-74941 Annecy, France. ${ }^{20}$ Department of Astronomy, The University of Tokyo, Bunkyo-ku, Tokyo, Japan. ${ }^{21}$ Department of Applied Physics, Fukuoka University, Jonan, Fukuoka, Japan. ${ }^{22}$ Faculty of Engineering, University of Toyama, Toyama, Toyama, Japan. ${ }^{23}$ Institute for Cosmic Ray Research (ICRR), Research Center for Cosmic Neutrinos (RCCN), The University of Tokyo, Kashiwa, Chiba, Japan. ${ }^{24}$ Yukawa Institute for Theoretical Physics (YITP), Kyoto University, Kyoto, Kyoto, Japan. ${ }^{25}$ Graduate School of Science, Osaka City University, Osaka, Osaka, Japan. ${ }^{26}$ JAXA Institute of Space and Astronautical Science, Sagamihara, Kanagawa, Japan. ${ }^{27}$ Institute for Cosmic Ray Research (ICRR), The University of Tokyo, Kashiwa, Chiba, Japan. ${ }^{28}$ Department of Physics, Ewha Womans University, Seoul, Korea. ${ }^{29}$ Department of Physics, Myongji University, Yongin, Korea. ${ }^{30}$ Department of Computer Simulation, Inje University, Gimhae, Korea. ${ }^{31}$ National Institute for Mathematical Sciences, Daejeon, Korea. ${ }^{32}$ School of Natural Science, Ulsan National Institute of Science and Technology (UNIST), Ulsan, Korea. ${ }^{33}$ Applied Research Laboratory, High Energy Accelerator Research Organization (KEK), Tsukuba, Ibaraki, Japan. ${ }^{34}$ Department of Physical Science, Hiroshima University, Higashi-Hiroshima, Hiroshima, Japan. ${ }^{35}$ Department of Physics and Institute of Astronomy, National Tsing Hua University, Hsinchu, Taiwan. ${ }^{36}$ Institute for Cosmic Ray Research (ICRR), Research Center for Cosmic Neutrinos 
(RCCN), The University of Tokyo, Hida, Gifu, Japan. ${ }^{37}$ California Institute of Technology, Pasadena, CA, USA. ${ }^{38}$ Institute for Advanced Research, Nagoya University, Nagoya, Aichi, Japan. ${ }^{39}$ Department of Physics, Hanyang University, Seoul, Korea. ${ }^{40}$ Korea Astronomy and Space Science Institute (KASI), Daejeon, Korea. ${ }^{41}$ National Center for High-Performance Computing, National Applied Research Laboratories, Hsinchu, Taiwan. ${ }^{42}$ Department of Physics, Tamkang University, New Taipei City, Taiwan. ${ }^{43}$ Department of Advanced Materials Science, The University of Tokyo, Kashiwa, Chiba, Japan. ${ }^{44}$ Istituto Nazionale di Fisica Nucleare, Sapienza University, Roma, Italy. ${ }^{45}$ The Institute of Statistical Mathematics, Tachikawa, Tokyo, Japan. ${ }^{46}$ School of Physics, Korea Institute for Advanced Study (KIAS), Seoul, Korea. ${ }^{47}$ Institute for Photon Science and Technology, The University of Tokyo, Bunkyo-ku, Tokyo, Japan. ${ }^{48}$ Disaster Prevention Research Institute, Kyoto University, Uji, Kyoto, Japan. ${ }^{49}$ Institute for Laser Science, University of Electro-Communications, Chofu, Tokyo, Japan. ${ }^{50}$ National Institute of Information and Communications Technology (NICT), Koganei, Tokyo, Japan. ${ }^{51}$ Department of Physics, Kyoto University, Kyoto, Kyoto, Japan. ${ }^{52}$ Faculty of Law, Ryukoku University, Kyoto, Japan. ${ }^{53}$ Department of Physics, University of Notre Dame, Notre Dame, IN, USA. ${ }^{54}$ Department of Physics, Wuhan Institute of Physics and Mathematics, CAS, Wuhan, China. ${ }^{55}$ The University of Shanghai for Science and Technology, Shanghai, China. ${ }^{56}$ Faculty of Engineering, Niigata University, Niigata, Niigata, Japan. ${ }^{57}$ Graduate School of Science and Technology, Niigata University, Niigata, Niigata, Japan. ${ }^{58}$ Center for Measurement Standards, Industrial Technology Research Institute, Hsinchu 30011, Taiwan.

${ }^{59}$ Department of Engineering, University of Sannio, Benevento, Italy. ${ }^{60}$ Faculty of Arts and Science, Kyushu University, Fukuoka, Fukuoka, Japan.

${ }^{61}$ Research Institute for Science and Engineering, Waseda University, Shinjuku-ku, Tokyo, Japan. ${ }^{62}$ National Institute of Technology, Nagaoka College, Nagaoka, Niigata, Japan. ${ }^{63} \mathrm{Kavli}$ Institute for the Physics and Mathematics of the Universe (IPMU), Kashiwa, Chiba, Japan. ${ }^{64}$ Nagaoka University of Technology, Nagaoka, Niigata, Japan. ${ }^{65}$ Graduate School of Science and Engineering, Hosei University, Koganei, Tokyo, Japan. ${ }^{66}$ Faculty of Science, Toho University, Funabashi, Chiba, Japan. ${ }^{67}$ Faculty of Information Science and Technology, Osaka Institute of Technology, Hirakata, Osaka, Japan.

${ }^{68} \mathrm{School}$ of High Energy Accelerator Science, The Graduate University for Advanced Studies (SOKENDAI), Tsukuba, Ibaraki, Japan.

${ }^{69}$ Istituto Nazionale di Fisica Nucleare, University of Perugia, Perugia, Italy. ${ }^{70}$ Faculty of Science, Niigata University, Niigata, Niigata, Japan.

${ }^{71}$ Department of Communications, National Defense Academy of Japan, Yokosuka, Kanagawa, Japan. ${ }^{72}$ Department of Physics, University of Florida, Gainesville, FL, USA. ${ }^{73}$ Department of Physics and Astronomy, Sejong University, Seoul, Korea. ${ }^{74}$ Department of Physics, Korea University, Seoul, Korea. ${ }^{75}$ Department of Astronomy, Beijing Normal University, Beijing, China.

*e-mail: chunglee.kim@ewha.ac.kr; michimura@granite.phys.s.u-tokyo.ac.jp; hisaaki.shinkai@oit.ac.jp; ayaka.shoda@nao.ac.jp 